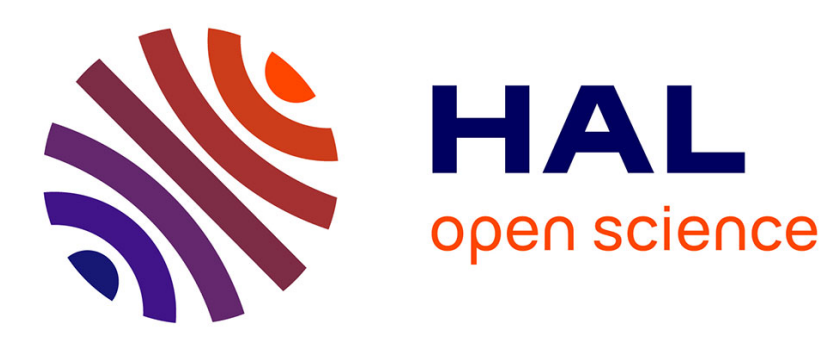

\title{
Coefficient of restitution interpreted as damping in vibroimpact
}

\author{
Kenneth Hunt, Erskine Crossley
}

\section{To cite this version:}

Kenneth Hunt, Erskine Crossley. Coefficient of restitution interpreted as damping in vibroimpact. Journal of Applied Mechanics, 1975, 10.1115/1.3423596 . hal-01333795

\section{HAL Id: hal-01333795 \\ https://hal.science/hal-01333795}

Submitted on 19 Jun 2016

HAL is a multi-disciplinary open access archive for the deposit and dissemination of scientific research documents, whether they are published or not. The documents may come from teaching and research institutions in France or abroad, or from public or private research centers.
L'archive ouverte pluridisciplinaire HAL, est destinée au dépôt et à la diffusion de documents scientifiques de niveau recherche, publiés ou non, émanant des établissements d'enseignement et de recherche français ou étrangers, des laboratoires publics ou privés. 


\section{Coefficient of Restitution Interpreted as Damping in Vibroimpact}

During impact the relative motion of two bodies is often taken to be simply represented as half of a damped sine wave, according to the Kelvin-Voigt model. This is shown to be logically untenable, for it indicates that the bodies must exert tension on one another just before separating. Furthermore, it denotes that the damping energy loss is proportional to the square of the impacting velocity, instead of to its cube, as can be deduced from Goldsmith's work. A damping term $\lambda x^{\prime \prime} \dot{x}$ is here introduced; for a sphere impacting a plate Hertz gives $n=3 / 2$. The Kelvin-Voigt model is shown to be approximated as a special case deducible from this law, and applicable when impacts are absent. Physical experiments have confirmed this postulate.

\author{
K. H. Hunt \\ Dean and Professor of Engineering, \\ Monash Universilty, \\ Clayton, Victorla, Australla \\ F. R. E. Crossley \\ Protessor, \\ Department of Mechanical Englneering. \\ University of Massachusetts, \\ Amherst, Mass.
}

\section{Introduction}

Section 1.1. This analysis is confined to compact solid bodies (normally regarded as elastic) impacting one another at fairly low velocities, e.g., below 20 ips $(50 \mathrm{~cm} / \mathrm{sec})$, for reasonable shapes of impacting profiles when the material is, say, hardened steel. No account is taken of the possible effect of reflected elastic shock waves on the forces in the zone of contact considered. Long rods impacted axially, and beams or plates impacted transversely, are therefore not regarded as "compact solid bodies" [1]," though it may be possible to incorporate the present conclusions as a part of a system which includes other vibratory effects as well.

Section 1.2. The classical definition of "coefficient of restitution" $e$ is, in simple one-dimensional impact between two rigid bodies in pure translation, the ratio of their relative speed after impact to that before, so that

$$
v_{0}=e v_{i},
$$

$v_{i}$ and $v_{0}$ being the relative speeds respectively "in" and "out." In [1] there is a good account of many experiments conducted with different materials, usually solid spheres, to determine values for $e$. Yet in these experiments, now rather antiquated, the effects of surface curvature on $e$ are not isolated. Nevertheless, for given impacting bodies $e$ invariably decreases as $v_{i}$ increases. For high $v_{i}$ one or both bodies can permanently deform plastically, but, as

\footnotetext{
1 Numbers in brackets designate References at end of paper.
}

stated in Section 1.1, this eventuality is not taken account of here, because the typical impact in the elastic range now to be analyzed is that which leaves both bodies unchanged in dimension and properties. Surely, then, one might conclude that, if the bodies are unchanged, $e$ must be unity and the impact perfectly reversible; but it need not he, because, while most of the elastic strain energy is restored, a proportion, which increases as $v_{i}$ increases, is dissipated in heat (random molecular vibrations) and, perhaps, in microscopic slip between grains if the material in question is crystalline. For a limited range of low $v_{i}$, and for most materials with a linear elastic range, $[1$, p. 258], it appears that one can write with tolerable accuracy

$$
e=1-\alpha v_{i}
$$

For steel, bronze, or ivory, $\alpha$ will have a value somewhere between 0.002 and $0.008 \mathrm{sec} / \mathrm{in}$. (i.e., $0.08-0.32 \mathrm{sec} / \mathrm{m}$ ), but the linear law of equation (2) cannot apply above some limiting value of $v_{i}$ which depends on the material and on the geometry of the surfaces at or near the zone of contact.

Section 1.3. If one of the bodies is taken to be stationary, the loss, over a total single-impact sequence, of the kinetic energy of the moving body (of mass $m$ ) is

$$
\Delta E=\frac{1}{2} m\left(v_{i}^{2}-v_{0}^{2}\right)
$$

and, using successively equations (1) and (2),

$$
\begin{aligned}
\Delta E & =\frac{1}{2} m v_{i}^{2}\left(1-e^{2}\right) \\
& =\frac{1}{2} m v_{i}^{2}\left\{1-\left(1-\alpha v_{i}\right)^{2}\right\} .
\end{aligned}
$$

Now, since $\alpha$ is much less than 1 (and because it is empirically determined we cannot expect perfect exactitude anyway), one may 


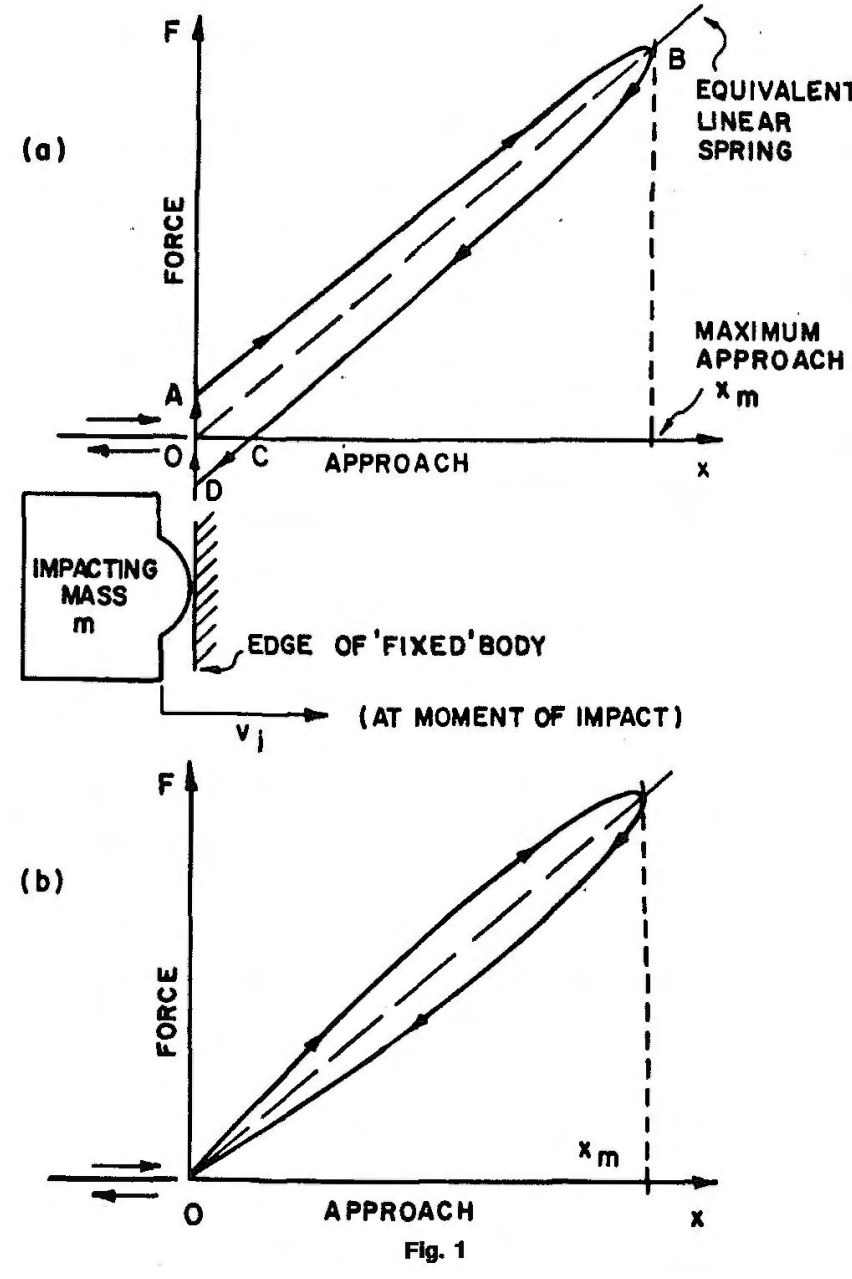

ignore $\alpha^{2} v_{i}{ }^{2}$ in comparison with $2 \alpha v_{i}$, and thus write with acceptable accuracy

or

$$
\Delta E=\frac{1}{2} m v_{i}^{2}\left(2 \alpha v_{i}\right)
$$

$$
\Delta E=\alpha m v_{i}^{3}
$$

Section 1.4. Some workers in vibroimpact, e.g., [2], and others concerned with impact dampers, for instance, have (sometimes with remarkable success) considered impact to be instantaneous, and have used a constant $e$ irrespective of $v_{i}$. In such work the "approach," namely, the extent by which the centers of mass of the two impacting bodies move toward and away from one another as a result of the localized deformation near the zone of contact, is ignored, and this practice may be justifiable when the interval of time between successive contacts is substantially longer than the duration of one contact. In [3], however, neither the time of contact nor the approach are ignored; the equation governing the period of contact during a single impact is taken to be a half period of damped harmonic vibration during which, in the absence of any other excitation, the standard form of linear equation

$$
m \ddot{x}+c \dot{x}+k x=0
$$

(in which $c$ is a constant coefficient of viscous damping and $k$ a linear spring-constant presumed to match the force-approach law) is taken to apply. In effect equation (4) matches precisely the ideal Kelvin-Voigt viscoelastic solid [4]. In the Appendix the consequences of the assumptions inherent in equation (4) are developed qualitatively, and the conclusions reached confirm the validity of the analysis which now follows.

Section 1.5. According to equation (4) the damping force, $c \dot{x}$, is greatest when $\dot{x}$ is highest, that is to say at the precise moment of impact when $\dot{x}=v_{i}$. This damping term $c \dot{x}$, and the spring term $k x$, can be combined to produce a form of hysteresis loop shown as $O A B C D O$ in Fig. 1(a), the shape of which is, as it turns out, close to a half ellipse, provided that the damping is small. This half-ellipse hysteresis loop is in conflict with the expected shape of a loop that arises from a compressive load being applied to and removed from a body within its elastic range at a slow rate. A loop of this more realistic form is sketched in Fig. 1(b). Because the applied load is compressive, through a pair of profiles that touch but are not otherwise joined together, no tensile force is admissible; i.e., since the "force" in Fig. 1(b) is everywhere compressive (namely, above the Ox-axis) the loop cannot be allowed to extend into the region below the Ox-axis. Moreover, the compressive force at the moment the load is applied is zero. These arguments lead to the form of hysteresis loop drawn in Fig. 1(b), the loop coming to a sharp point at the origin. The half ellipse in Fig. 1(a), that arises simply from the direct intrusion of equation (4), is sufficiently different from the loop of Fig. 1(b) to raise doubts about its validity as a model of what actually occurs, because $(a)$, for the region $0<x$ $<\mathrm{OC}$ of the load-removal portion, it hypothesises a tensile force (which would tend to lengthen the period of contact and reduce the speed at which the impacting body leaves), and (b), at the moment of impact, there is a "shock" application of a supposed force that arises entirely from the damping term $c \dot{x}$ in equation (4).

Section 1.6. True, when the impacting bodies are highly resilient (namely, when $c$ is very much less than the critical damping coefficient), these two anomalous effects may be so small as to cause no concern to someone establishing a mathematical model suitable for analogue or digital simulation. It is however demonstrated below, equation (16), that a much better model can quite easily be set up which, in contrast to the one just described, shows promise of being usefully adaptable both to bodies with nonlinear elastic properties and to coefficients of restitution substantially less than unity.

Section 1.7. Fig. 1(a) fails in another respect to meet the required conditions; the half-ellipse hysteresis loop remains to all intents and purposes identically proportioned with respect to the rest of the diagram whatever the maximum approach $x_{m}$. The area of the loop, which represents the energy loss $\Delta E$, would then (for resilient bodies) be closely proportional to $x_{m}{ }^{2}$, which, since

$$
\frac{1}{2} m v_{i}^{2} \approx \frac{1}{2} k x_{m}^{2}
$$

may be taken as being proportional to $v_{i}{ }^{2}$. Yet equation (3) requires $\Delta E$ to be much more nearly proportional to $v_{i}{ }^{3}$ than to $v_{i}{ }^{2}$.

Section 1.8. Equation (4) has the advantage of simplicity. As it stands, for an isolated half period of impact-duration, it is linear, and in the well-known classical form. But, since the overall pattern of impact (including relative movements across clearances, etc.) is far from linear-at best it is piecewise linear-one may justifiably propose some improvement on the highly idealized Kelvin-Voigt model, particularly now that there are available simulation techniques that readily accept nonlinearities and still give high accuracy. Clearly the hysteresis loop in Fig, 1(a) should be abandoned and replaced by one looking more like that in Fig. 1(b). Its shape should be consistent with damping forces being zero both when $x$ $=0\left(\dot{x}=v_{i}\right.$ or $\left.\dot{x}=-v_{0}\right)$ and when $x=x_{m}(\dot{x}=0)$. A damping term of the form $\lambda(x)^{p}(\dot{x})^{q}$ satifies these boundary conditions, and a way is described next for determining suitable values for $\lambda, p$, and $q$.

Section 1.9. The force-approach law based on the Hertzian theory for localized normal and frictionless contact between linearly elastic bodies is not itself linear (as required by equation (4)) but is nonlinear and of the form $F=k x^{3 / 2}$. The theory, described in $[1,5]$ and by many other authorities, cannot be regarded as valid unless the zone of contact is of small dimensions in comparison with the radii of curvature of the impacting bodies in its vicinity, 
and unless it has very large radii of curvature in comparison with its linear dimensions. For two bodies of steel (elastic modulus $30 \times$ $10^{6} \mathrm{lb} / \mathrm{in.}^{2}\left(2.068 \times 10 \mathrm{~N} / \mathrm{m}^{2}\right)$ and Poisson's ratio 0.30$)$ impacting in spherical regions whose radii of curvature are $R_{1}$ and $R_{2}$, the forceapproach law becomes

$$
F=K_{1} x^{3 / 2}\left(\frac{1}{R_{1}}+\frac{1}{R_{2}}\right)^{-2}
$$

where $K_{1}=2.19 \times 10^{7}$ if $R_{1}, R_{2}$ and $x$ are measured in in. and $F$ in $\mathrm{lb}$, or $K_{1}=3.83 \times 10^{6}$ if $R_{1}, R_{2}$, and $x$ are measured in $\mathrm{cm}$ and $\mathrm{F}$ in newtons. The maximum shear stress (occurring at points on the common normal a little below the surfaces of the bodies) is of value

$$
\tau=K_{2} \sqrt[3]{\left(\frac{1}{R_{1}}+\frac{1}{R_{2}}\right)^{2} F}
$$

$K_{2}$ being $1.16 \times 10^{4}$ if $\tau$ is to be read in lb/in. ${ }^{2}$; or $K_{2}=9.02 \times 10^{7}$ for $r$ in $\mathrm{N} / \mathrm{m}^{2}$. In [1 and 5], and elsewhere, it is explained how equations (5) and (6) can be formulated for more general shapes of contacting contours, and the results here presented can with complete validity be extended if desired.

Section 1.10. According to [6], direct compressive contact stresses (of a highly localised nature) exceeding $200,000 \mathrm{lb} / \mathrm{in}^{2}$ $\left(1.38 \times 10^{9} \mathrm{~N} / \mathrm{m}^{2}\right)$ are not uncommon for conditions of continuous operation in rolling bearings, and in certain circumstances an acceptable limit may be as high as $500,000 \mathrm{lb} / \mathrm{in} .{ }^{2}\left(3.45 \times 10^{9} \mathrm{~N} / \mathrm{m}^{2}\right)$. For ductile materials [1] the most probable form of impact failure is in shear and, according to the Hertzian theory, maximum shear is about 0.31 times the maximum compressive stress, this factor being used in writing equation (6). So, in view of the foregoing, a limiting value $\tau_{\text {lim }}$ of $0.31 \times 200,000=62,000 \mathrm{lb} / \mathrm{in}^{2}\left(4.27 \times 10^{8}\right.$ $\mathrm{N} / \mathrm{m}^{2}$ ) appears to be reasonable, even conservative, for alloy steels with surface properties suitable not only for rolling bearings, but also for other joint-pairs with highly localized regions of contact such as those often found in cams, gears, etc. This limiting localized shear-stress value is, incidentally, 10 times higher than the unrealistically low limit used in part of [3], and it is seen below to be reasonably compatible with impact speeds of the magnitudes mentioned in Section 1.1.

Secion 1.11. The 3/2-power law of equation (5) does not apply when, for instance, contact is between perfectly flat surfaces; then, at least ideally, the area of the zone of contact does not change during the time of impact, and a linear force-approach law would probably apply. For parallel cylinders in longitudinal contact along a narrow zone on either side of a line, an index somewhere between 1 and 1.5 could be expected to match the conditions fairly well, and this does not conflict greatly with various formulas to be found in $[1,3,6,7,9,10]$. Harris $[6]$ emphasises that, unless conditions are very special, a uniform force per unit length of roller cannot be assumed. In any case a formula for cylinders in longitudinal contact must depend for its validity on extremely precise alignment and highly exact knowledge of the axial distribution of the applied load; even in precision engineering applications information on these two matters may be hard, or impossible, to guarantee. So, despite the doubts about these special geometries yielding valid formulas, a more general form of the force-approach law of equation (5), namely,

$$
F=k x^{n}
$$

will be used in what follows, and it will be found that it is possible to use a variable damping coefficient in an equation derived by extension and generalization of equation (4). It is then seen that the damping factor $\lambda$ (see Section 1.8) can be made independent of the index $n$ in equation (7).

\section{Analytical Formulation}

Section 2.1. Consider first nonlinear elastic impact $(e=1)$ of a mass $m$ with an impacting speed $v_{i}$ Fig. 2. The kinetic energy at contact is $(1 / 2) m v_{i}^{2}$, and, provided next that the proposition of
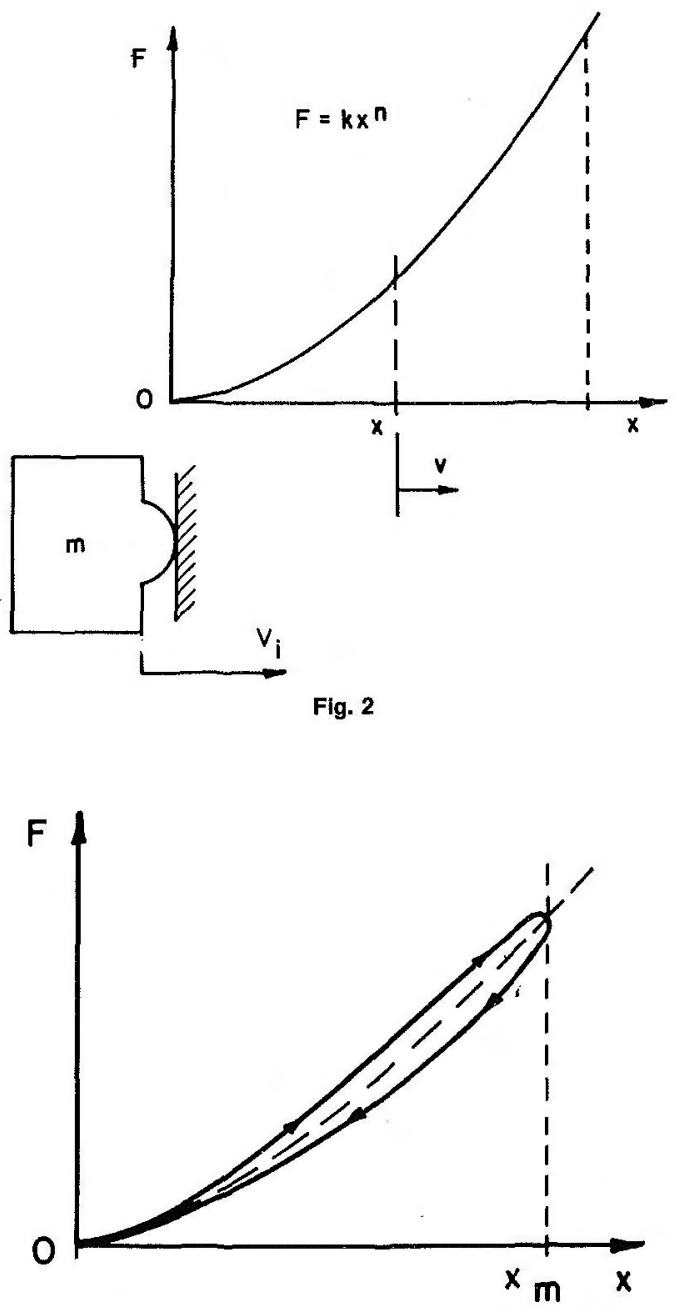

Fig. 3

small energy dissipation holds, this can be equated with reasonable accuracy, via equation (7), to the maximum elastic-strain energy stored, i.e., for a maximum approach $x_{m}$,

$$
\frac{1}{2} m v_{i}^{2}=\int_{0}^{x_{m}} F \mathrm{~d} x=k \int_{0}^{x_{m}} x^{n} d x
$$

or

$$
\frac{1}{2} m v_{i}^{2}=\left(\frac{k}{n+1}\right) x_{m}^{(n+1)}
$$

and

$$
v_{i}=\sqrt{\frac{2 k}{m(n+1)}} x_{m}^{\frac{(n+1)}{2}} .
$$

For an intermediate position $x, 0<x<x_{m}$,

$$
\frac{1}{2} m v^{2}=\frac{1}{2} m v_{i}^{2}-\int_{0}^{x} F d x
$$

and

$$
v=\sqrt{\frac{2 k}{m(n+1)}} \sqrt{x_{m}^{(n+1)}-x^{(n+1)}} .
$$

Section 2.2. Now in fact there is an (assumed relatively small) hysteretic energy loss represented by the area of a hysteresis loop, Fig. 3. This area can be equated to $\Delta E$ and, combining equations (3) and (9),

$$
\Delta E=\alpha m v_{i}^{3}=\frac{\alpha}{\sqrt{m}}\left(\frac{2 k}{n+1}\right)^{3 / 2} \dot{x}_{m}^{\frac{3(n+1)}{2}} .
$$


If the notion of a nonconstant damping coefficient is accepted, one may also write, assuming $q=1$ (see Section 1.8)

$$
E=\oint c v d x
$$

where the $\varnothing$ refers to the circuit around the loop in Fig. 3. Now, with an energy loss assumed to be small, equation (10) can be used with fair accuracy to give $v$ and, since the loop's area is shared sufficiently equally between "inward" and "outward" paths, the cyclic integral can be written

$$
2 \int_{0}^{x_{m}} \operatorname{cvdx}
$$

which in turn leads to

$$
\Delta E=2 \sqrt{\frac{2 k}{m(n+1)}} \int_{0}^{x_{m}} c \sqrt{x_{m}^{(n+1)}-x^{(n+1)}} d x
$$

The next question is how to evaluate this integral to produce the nonconstant $c$ in managable form. This question can quite easily be resolved if one introduces the constant $\lambda$ (see Section 1.8, making $p=n$ ), and thus writing

$$
c=\lambda x^{n} .
$$

Then equation (12) becomes integrable and reduces to

$$
\Delta E=\frac{2 \lambda}{\sqrt{m}} \sqrt{\frac{2 k}{n+1}}\left(\frac{1}{n+1}\right)\left(\frac{2}{3}\right) x_{m}^{\frac{3(n+1)}{2}}
$$

This expression for $\Delta E$ is required to be the same as that of equation (11). Thus one reaches the extremely simple conclusion that

$$
\lambda=\frac{3}{2} \alpha k \text {. }
$$

Note that, as anticipated in Section 1.11, equation (15) does not contain the index $n$. Using it with an $n$th power force-approach law, the equation (applicable to a free damped half cycle of vibroimpact) is now not equation (4) but

$$
m \ddot{x}+\left(\lambda x^{n}\right) \dot{x}+k x^{n}=0
$$

or

$$
m \ddot{x}+k x^{n}\left(\frac{3}{2} \alpha \dot{x}+1\right)=0 .
$$

The index $q$ (see Section 1.8) need not be taken as unity; several suitable choices could be made so long as they are compatible with equation (12) being easily integrable and leading to an expression in which $x_{m}$ has a power of

$$
\frac{3(n+1)}{2} .
$$

For instance if $q=2, \lambda$ comes out to

$$
\frac{3}{8} \alpha \sqrt{2 k m(n+1)}
$$

then instead of the second of equations (16) one has

$$
m \ddot{x}+k x^{n}\left\{\left(\frac{3}{8} \alpha \sqrt{2 \frac{m}{k}(n+1)}\right) \dot{x}^{2}+1\right\}=0,
$$

the damping factor $\lambda$ being no longer independent of $n$.

\section{Closure}

Section 3.1. The authors have found it quite simple to incorporate equation (16) in digital computer simulation of a forced vibroimpact system, and in some instances the response is significantly at variance with that of an equivalent linearized model. Some specimen hysteresis loops, obtained from trial runs, are presented in Fig. 4. These all apply to a steel body weighing about one
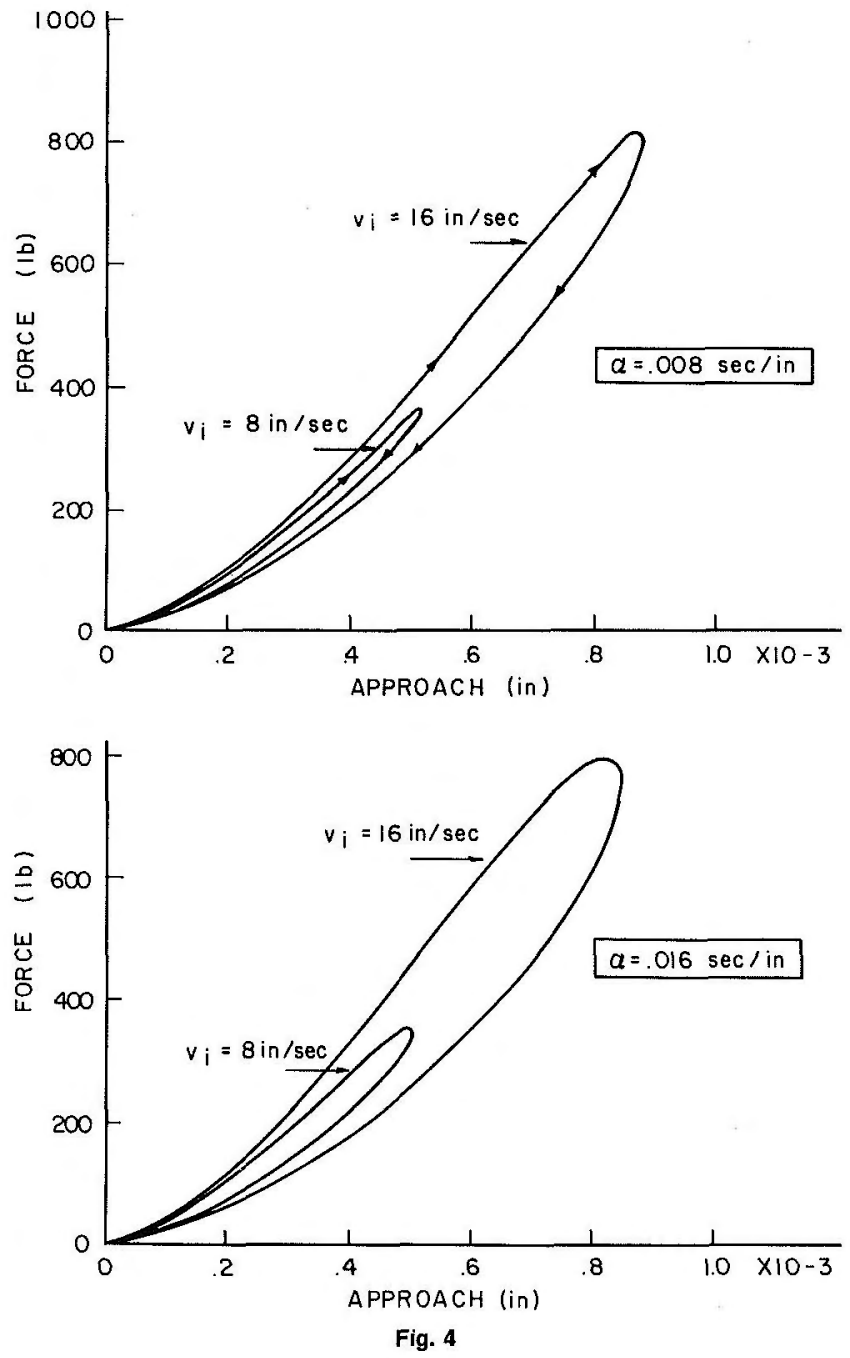

pound (4.448 N) having a convex spherical surface of radius $R_{1}=1$ in. $(2.54 \mathrm{~cm})$ impacting a concave spherical steel surface of radius $R_{2}=-2$ in. $(-5.08 \mathrm{~cm})$. The undamped force-approach law, equation (5), then becomes

$$
F=K_{3} x^{3 / 2}
$$

with $K_{3}=3.1 \times 10^{7}$ if $x$ is in in., $F$ in lb, or $K_{3}=3.4 \times 10^{7}$ for $x$ in $\mathrm{cm}, F$ in newtons $\left(4.27 \times 10^{8} \mathrm{~N} / \mathrm{m}^{2}\right)$ and, corresponding with a shear stress of $62,000 \mathrm{lb} / \mathrm{in}^{2}$ (see Section 1.10), the maximum allowable force, by equation (6), is $600 \mathrm{lb}(2690 \mathrm{~N})$. As can be seen from Fig. 4, when the impacting speed is 16 ips $(40 \mathrm{~cm} / \mathrm{sec})$ this figure of $600 \mathrm{lb}$ is exceeded, but the shear stress (equation (6)) is proportional to the cube root of normal force, and the figure of 62,000 $\mathrm{lb} / \mathrm{in}^{2}\left(4.27 \times 10^{8} \mathrm{~N} / \mathrm{m}^{2}\right)$ is not exceeded by more than about 15 percent. With the same impacting speed the shear stress can be reduced by altering the surface-geometries, e.g., by wrapping the concave surface more around the convex impacting mass. However, if the two impacting surfaces were to match one another too closely, the Hertzian analysis would no longer be valid (see Section 1.9 , particularly the italicized portion); for this reason much of the modeling described in [3] cannot be applied to this form of impactpair.

Section 3.2. Hysteresis loops similar to those in Fig. 4 have been drawn for $\alpha=0.002,0.004,0.008$, and $0.016 \mathrm{sec} / \mathrm{in}$. As mentioned in Section 1.2, values of $\alpha$ somewhere between 0.002 and $0.008 \mathrm{sec} / \mathrm{in} .(0.08-0.32 \mathrm{sec} / \mathrm{m})$ appear to be reasonable. For $\alpha$ to have a value as low as 0.002 the material would have to be particularly resilient. For a material with $\alpha$ as large as $0.016 \mathrm{sec} / \mathrm{in} .(0.64$ $\mathrm{sec} / \mathrm{m}$ ) the hysteresis loop may, with large $v_{i}$, become so large as to 
call into question several of the assumptions made in the derivation of equation (15). Nevertheless, a hysteresis loop of moderately convincing shape is, even then, automatically produced, and it is tentatively put forward that the method here described might be usefully applied in modeling situations quite far removed from those initially regarded as applicable.

Section 3.3. The whole proposition here put forward devolves in the first place on the validity of equation (2), which in turn is supported by the evidence of many experimenters who worked with a wide variety of apparatus over the best part of a century; see the bibliography in reference [1]. Even so one must not ignore the present need for up-to-date verification and extension of the concept of coefficient of restitution to new engineering materials. New experiments must not neglect, as earlier workers appear to have done, the dependence of coefficient of restitution on the geometry of the impacting surfaces. Also any experiment must be carefully contrived so that the cycle of impact is as simple as possible, so that there is no risk of making circuits of one or more smaller hysteresis loops within the main one during a single impact. The authors suspect that this may have given rise to discrepancies in past experiments, and indeed that it accounts for extraordinarily low coefficients of restitution quoted in [2]. Even though the notion of coefficient of restitution is inexact, the analysis here pursued shows that, in an engineering context at least, there is much to recommend it, and there is scope for further refinement of it.

\section{Acknowledgments}

The criticisms and helpful comments of Prof. J. D. C. Crisp (Monash University) are much appreciated. The assistance of the National Science Foundation through Grant GK-32372 is gratefully acknowledged.

\section{References}

1 Goldsmith, W. Impact, Edward Arnold Ltd., London, 1960.

2 Kobrinskii, A. E., Mechanisms With Elastic Connections and Impact Systems (translated from the Russian by R. Lennox-Napier), Iliffe Books Ltd., London, 1969.

3. Dubowsky, S., and Freudenstein, F., "Dynamic Analysis of Mechanical Systems With Clearances, Part 1: Formation of Dynamic Model; Part 2. Dynamic Response," Journal of Engineering for Industry, TRANS. ASME, Vol. 93, Series B, Feb. 1971, pp. 305-316.

4 Flügge, W., Viscoelasticity, Blaisdell Publishing Company, Waltham, Mass., 1967.

5 Timoshenko, S. P., and Goodier, J. N., Theory of Elasticity, McGraw-Hill, New York, 1970.

6 Harris, T. A., Rolling Bearing Analysis, Wiley, New York, 1966.

7 Timoshenko, S. P., and Baud, R. V., "The Strength of Gear Teeth," Mechanical Engineering, Vol. 48, No. 11, Nov. 1926, pp. 1105-1109.

8 Buckingham, E., Analytical Mechanics of Gears, McGraw-Hill, New York, 1949.

9 Lubkin, J. L., Handbook of Engineering Mechanics, ed., Flügge, W., McGraw-Hill, New York, Chapter 42, 1962.

10 Hugk, H., Nerge, G., and Stange, H., "Untersuchung der Kontaktschwingungen an der Eingriffsstelle von Kurvenmechanismen," Maschinenbautechnik, Vol. 16, June 1967, pp. 295-301.

\section{APPENDIX}

Suppose a perfectly elastic body of mass $m$ is in the gap between two perfectly elastic massive blocks leaving an overall clearance $g$. If the two compressive force-approach laws (to left and right) are both taken to be linear, the combined piecewise linear force-approach law is as shown in Fig. 5(a).

Now with the gap closed up to be just zero, the body in its central position just touches both blocks (with zero compressive forces on both sides), and the combined force-approach law is as in Fig. 5(b). The central horizontal portion of Fig. 5(a) has vanished, and the two linear end sections line up with one another exactly.

The force-approach curve of Fig. 5(b) is, then, identical in shape with one that would result if the body were securely fastened to one of the blocks, the other block being removed, Fig. 5(c). Now, however, when the approach $x$ is negative, the force is not compressive acting on the left of the body but tensile acting on the right.

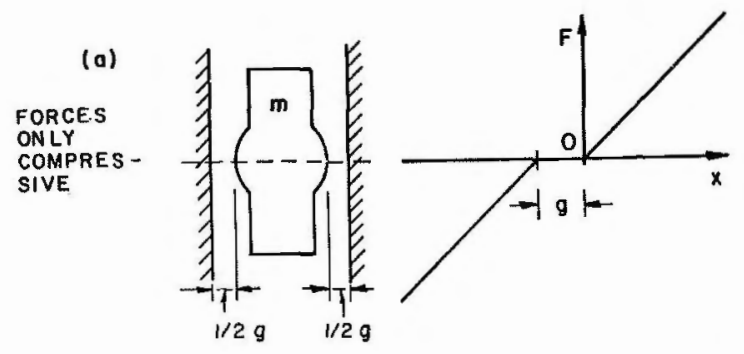

(b)

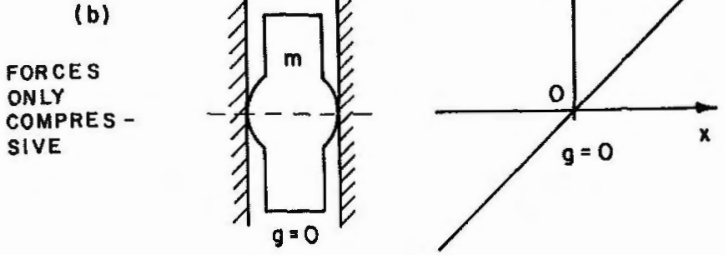

(c)

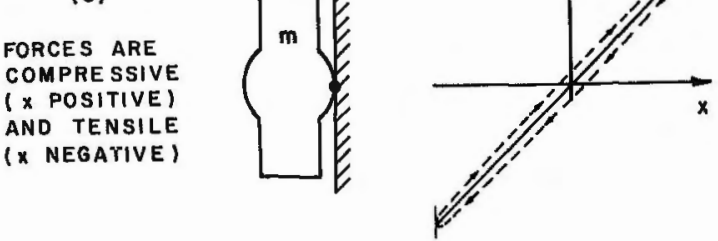

Flg. 5

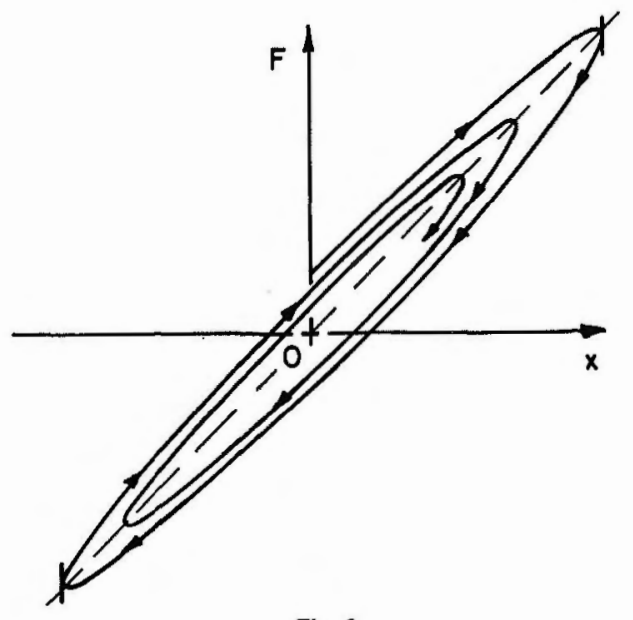

Fig. 6

For free vibrations of the system in Fig. $5(\mathrm{c})$ the analogy with the classical spring-mass system immediately springs to mind. If, in actuality, a small amount of energy is dissipated each cycle in friction, why should one not reasonably postulate a thin hysteresis loop, roughly elliptical, spreading a little on either side of the original (nondissipative) force-approach line? This loop would steadily diminish in size (exponentially) corresponding with the exponential decay of the amplitude in the classical theory of damped vibrations, Fig. 6. And this common assumption is known to apply well in many instances of mechanical vibrations. But is it valid to superpose such a loop on the, apparently identical, linear force-approach line in Fig. $5(b)$ ? The theme of the present paper is that such superposition is dubious, because the instance of Fig. 5(b) entails two impacts per cycle, whereas there are no impacts at all for Fig. $5(c)$. There is discontinuity between the positive and negative portions of Fig. $5(b)$, the body being in compressive contact first 


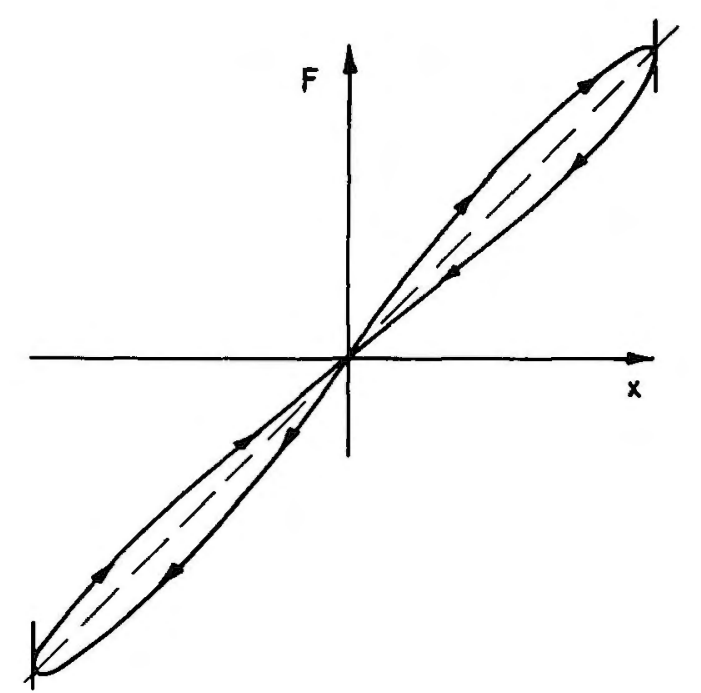

Fig. 7

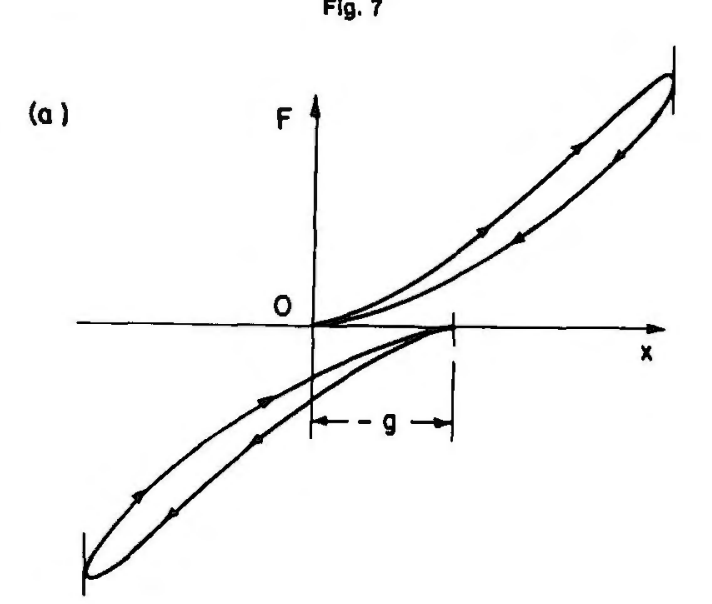

(b)

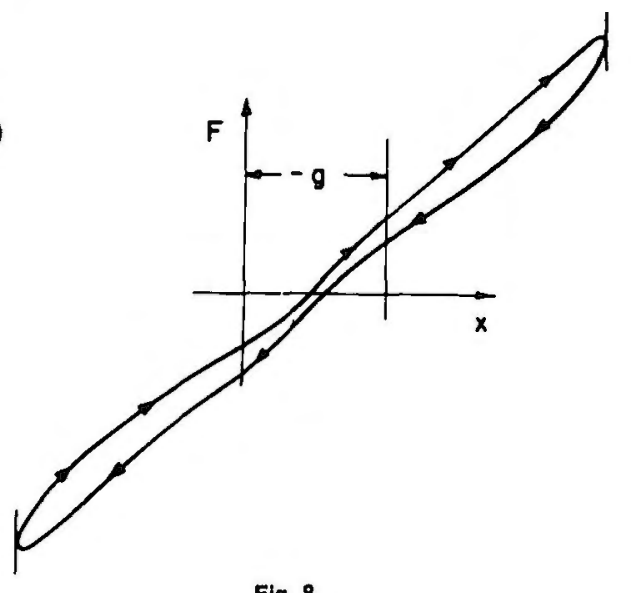

Fig. 8

on its right side, returning to its central position, and then, at the instant when it leaves the right-hand block, impacting compressively the left-hand block. The force-approach hysteretic law applicable to one cycle of the instance of Fig. $5(b)$ is, when small quantities of energy are lost, far more realistically represented by Fig. 7. 'I'he left and right-hand halves of Fig. 7 can be separated by a distance $g$ and then applied as they stand to Fig. 5(a) with more authenticity than two separated half ellipses, fig. 1(a).

When the force-approach law is nonlinear (as in Fig. 2), two loops of the form in Fig. 3 can be used on either side of a gap, and this has been the hasis of the modeling mentioned in Section 3.1. It is illuminating to see what happens if the gap $g$ is made negative, the body, when in its central position, now being under equal and opposite compressive force on either side. 'Then the two loops of

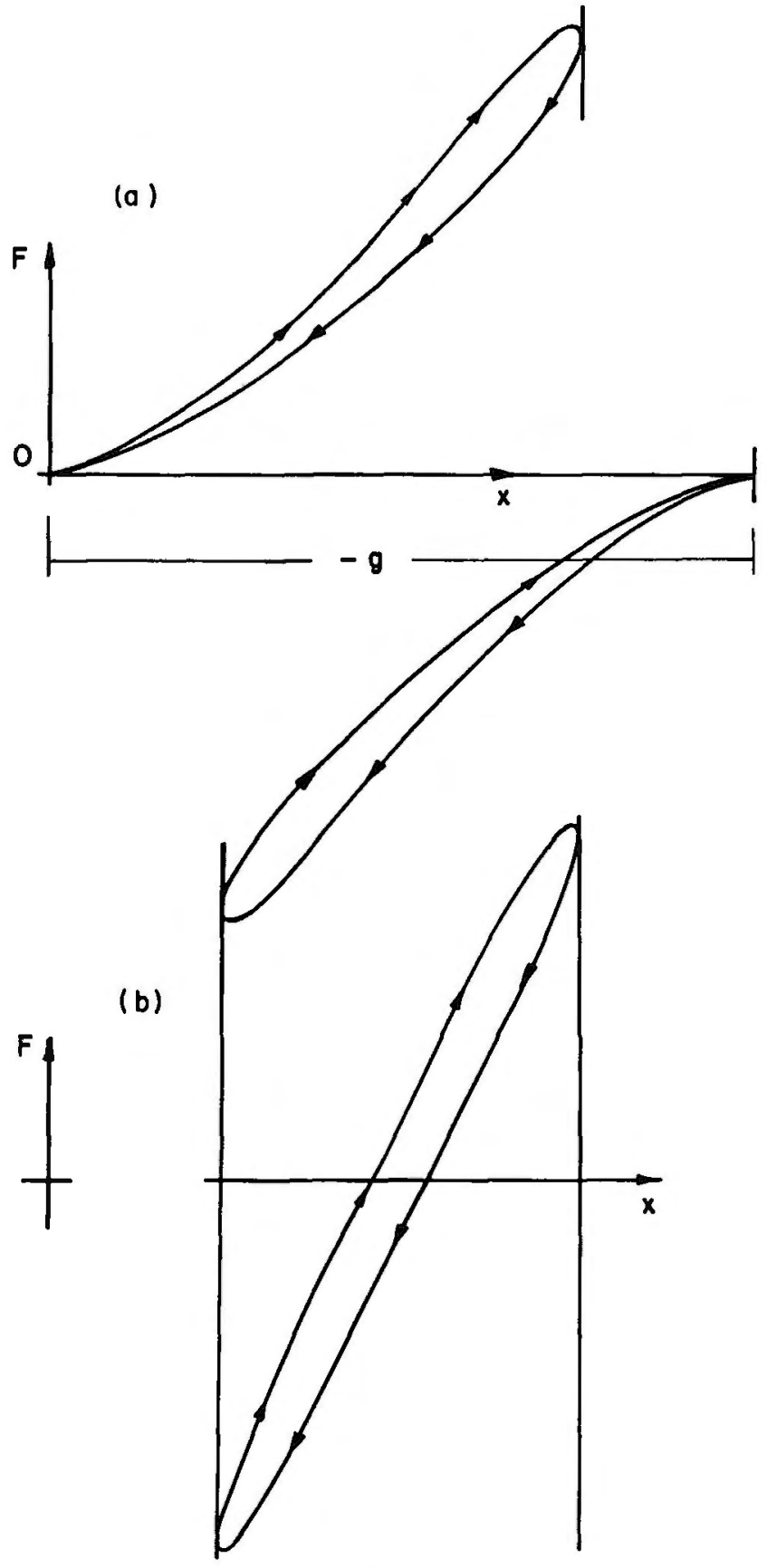

Fig. 9

the form in Fig. 3 overlap, Fig. 8(a). The resultant (i.e., the sum) of these two lonps (for a complete double-impact cycle) is drawn in Fig. $8(b)$. Because the amplitude here is greater than $1 / 2|g|$ there is still impact on both sides. When the amplitude is less than $1 / 2 \mathrm{dg}$ it may be expected that something like Figs. 9(a) and (b) applies. Now, of course, there is no impact at all, the body being firmly sandwiched between the blocks all the time, and the resultant loop of Fig. $9(b)$ begins to look much more like the expected elliptical shape used as a basis for Fig. 6, and indeed supports the validity of the classical approach via a Kelvin-Voigt model in situations where there is no impact at all.

The qualitative line of reasoning put forward in this Appendix therefore links the nonimpacting with the impacting situations and, though distinct from the main part of the paper, supports the analysis which is used in Section 2 to derive suitable differential equations for models that are considered to relate acceptably closely to reality. 